A N N A L E S Annales de Bretagne et des Pays de l'Ouest

Anjou. Maine. Poitou-Charente. Touraine

111-4 | 2004

Varia

\title{
Une affaire de congément à Botsorhel
}

La protestation convenancière au début de la Révolution

\section{Alain Le Bloas}

\section{(2) OpenEdition}

Journals

Édition électronique

URL : http://journals.openedition.org/abpo/1168

DOI : 10.4000/abpo.1168

ISBN : 978-2-7535-1496-6

ISSN : 2108-6443

Éditeur

Presses universitaires de Rennes

Édition imprimée

Date de publication : 20 décembre 2004

Pagination : $7-27$

ISBN : 978-2-7535-0082-2

ISSN : 0399-0826

Référence électronique

Alain Le Bloas, "Une affaire de congément à Botsorhel », Annales de Bretagne et des Pays de l'Ouest [En ligne], 111-4 | 2004, mis en ligne le 20 décembre 2006, consulté le 01 mai 2019. URL : http:// journals.openedition.org/abpo/1168; DOI : 10.4000/abpo.1168 


\title{
Une affaire de congément à Botsorhel
}

\author{
La protestation convenancière \\ au début de la Révolution
}

\author{
Alain LE BLOAS \\ professeur d'histoire-géographie - collège de l'Iroise, Brest
}

Depuis un siècle, l'historiographie révolutionnaire n'a cessé de s'intéresser à la question agraire ${ }^{1}$. Léon Dubreuil, le premier, a montré qu'en Basse-Bretagne cette dernière avait pour nom domaine congéable ${ }^{2}$. Malheureusement, ses recherches pionnières sur la question, engagées au début du $\mathrm{XX}^{\mathrm{e}}$ siècle, n'ont guère suscité de suites. Le combat des domaniers bretons pendant la Révolution est ainsi retombé dans l'oubli. Pourtant il fut aussi massif et déterminé que celui des autres paysans français. Outre l'absence de recherche récente sur le sujet - à l'exception toutefois de la thèse de Christian Kermoal qui y consacre quelques pages ${ }^{3}$-, la raison de cet oubli tient certainement aux moyens utilisés pour mener la lutte, à savoir le droit de pétition et les élections, pendant que le reste du pays, à commencer par la Haute-Bretagne, était régulièrement secoué par de véritables jacqueries. Les domaniers bretons n'ont dérogé à leur légalisme qu'à quelques rares occasions, et encore sans jamais atteindre le niveau de vio-

1. ADo, Anatoli, Paysans en révolution. Terre, pouvoir et jacqueries 1789-1793, Paris, 1996, p. 9-27; BIARD, Michel et BIANCHI, Serge, « La terre et les paysans pendant la Révolution française. Une orientation bibliographique ", Annales Historiques de la Révolution Française, ${ }^{\circ} 315$, janvier-mars 1999, p. 163-182.

2. Léon Dubreuil a rencontré la question du domaine congéable lors de sa thèse, $L a$ vente des biens nationaux dans les Côtes-du-Nord (1780-1800), Paris, 1912. Il lui a ensuite consacré un ouvrage spécifique, Les vicissitudes du domaine congéable en BasseBretagne à l'époque de la Révolution, Rennes, 1915, 2 vol. Avant Léon Dubreuil, la contestation convenancière avait une première fois été mise en évidence par Henri Sée, dans Les classes rurales en Bretagne du XVI siècle à la Révolution, Brionne, 1978 (reprint), et dans Cahiers de doléances pour la sénéchaussée de Rennes pour les états généraux de 1789, Rennes, 1912, vol. IV, ainsi que par Philippe Sagnac et Pierre Caron, dans Les Comités des droits féodaux et de législation et l'abolition du régime seigneurial (17891793), Paris, 1907.

3. Kermoal, Christian, Les Notables paroissiaux du Trégor. Permanences et évolutions (1770-1850), Thèse, Rennes 2, 2000, 4 vol. dactyl., plus spécialement I p. 60 et II p. 444 448 , p. 476-481, p. 485-491. 
lence rencontré ailleurs ${ }^{4}$. Parmi ces quelques exemples, il y a la mobilisation des habitants de Botsorhel en vue d'empêcher le déroulement d'un congément à la fin de l'année 1790 .

Construire un récit et lui donner du sens en partant des quelques lignes constituant un arrêté de l'administration départementale n'a pas été chose aisée. À partir de cette trace, c'est à un véritable jeu de piste, presque à une enquête policière qu'il a fallu se livrer. Des dizaines de liasses et registres ont été consultées pour en extraire un chiffre, une phrase, un paragraphe, et parfois un document entier... Ces difficultés sont inhérentes à toute recherche de type micro-historique. Cette dernière démarche nous semblait, en effet, pour l'instant la plus susceptible de fournir des réponses à certaines questions. Quelle est la réception réelle du domaine congéable à la fin du XVIII ${ }^{\mathrm{e}}$ siècle? Comment circule l'information dans les campagnes? Quels sont les canaux, les modalités, les réseaux et les leaders de la mobilisation convenancière? À quel rythme obéit-elle? Quelle est sa géographie? Quels sont ses objectifs et comment évoluent ces derniers dans le temps? Quelle est la position des nouvelles administrations mises en place en 1790, depuis les communes jusqu'au département, et le rôle joué par chacune pour accélérer ou au contraire freiner le mouvement? Cette formule possédait à nos yeux un autre avantage : mettre sur le devant de la scène les simples paysans et non pas seulement les plus riches d'entre eux et leurs alliés bourgeois.

\section{La terre et les hommes à Botsorhel à la fin du XVIII ${ }^{\mathrm{e}}$ siècle}

La paroisse de Botsorhel est située dans le Trégor finistérien ou Petit Trégor, au pied des rudes monts d'Arrée. Ogée la décrit ainsi : "Ce territoire, arrosé par la rivière du Ponthou, qui le traverse, est assez fertile en grains, mais la majeure partie est en landes, montagnes et bois, dont le plus considérable est celui de Béfou, d'environ une lieu et un quart de périmètre ${ }^{5}$. " En 1789, la paroisse compte à peu près 160 feux, ce qui donne 889 habitants lors du recensement de $1790^{6}$. Le rôle des vingtièmes établi pour l'année 1790 nous fournit une photographie de la propriété foncière et des modes de faire-valoir utilisés à Botsorhel ${ }^{7}$. S'y trouvent recensés 74 convenants, 3 métairies, 4 censives, 52 autres biens fonciers très souvent loués à domaine congéable (comme pour les convenants, le rôle opère alors une distinction entre le fonds et les droits superficiaires). Comme on le voit, la terre est alors presque entièrement acconvenancée. Les moulins le sont aussi en partie (au moins deux sur sept).

4. DuBReull, Léon, « La lutte économique au début de la Révolution. Une affaire de congément à Loguivy-Plougras ", dans La Révolution dans le département des Côtes-duNord, Paris, 1909, p. 67-103.

5. OGEE, Dictionnaire historique et géographique de la province de Bretagne, Rennes, 1853 (rééd.), I, p. 95.

6. Arch. dép. du Finistère, 10 L 164.

7. Ibidem, $13 \mathrm{~L} 12$. 
Qui sont les propriétaires des domaines congéables? Les plus gros fonciers sont nobles, à commencer par Etienne René Callouët de Lanidy ${ }^{8}$. Ce dernier, sur le territoire de Botsorhel, possède un manoir, 32 convenants, 2 grosses métairies (dont celle de Keraël, où se trouve le manoir), 12 autres biens fonciers, une maison au bourg, une dîme sur 6 convenants, 3 moulins, ainsi que le fief de Keralliou. Sa taxation aux vingtièmes et fouages est de 401 livres, 10 sous et 3 deniers, soit 18,3\% de la somme totale due par les contribuables de Botsorhel. Sa capitation est de 321 livres, 11 sous et 3 deniers (dont 7 livres pour les domestiques), soit 59,2\% de l'imposition de la paroisse ${ }^{9}$. Les autres seigneurs fonciers (principalement Castellan et Mouillemuse) viennent très loin derrière. Quant à la propriété ecclésiastique, elle est marginale.

La série Q permet d'en savoir davantage sur ces domaines congéables et le fonctionnement du système convenancier à Botsorhel ${ }^{10}$. Sur les 31 biens de première et seconde origine vendus dans la commune, au moins 17 sont des domaines congéables. L'échantillon est réduit et manque de représentativité vu l'absence des biens du principal propriétaire, par ailleurs protagoniste essentiel du congément sujet de cette recherche ${ }^{11}$.

Le convenant type a une superficie comprise entre 5 et 15 ha. Il dépasse rarement cette taille. Celui de Kergariou atteint bien 34 ha, mais, selon les experts chargés de l'évaluer, c'est le plus beau du canton de Guerlesquin. Le domaine congéable de Saint-Esser, lui, fait presque 18 ha mais semble surtout constitué de terres froides. Autant que la taille, ce qui fait la valeur d'un bien c'est en effet l'importance des terres chaudes (les plus fertiles) et la complémentarité des parcelles (terres chaudes + terres froides + prés). Le boisement du convenant, soit de la surface (3 cas seulement), soit des fossés, soit des deux, le bonifie. On remarquera à ce propos, avant de l'expliquer plus loin, la pauvreté en bois de la plupart des convenants, y compris sur les fossés. Bien évidemment, ce qui donne aussi sa valeur à un domaine congéable ce sont les édifices (bâtiments, talus, fossés) construits, entretenus et améliorés par le domanier. D'ailleurs les procès-verbaux d'expertise les décrivent toujours, même si les droits réparatoires restent la propriété du domanier.

Les informations relatives au prélèvement sont légèrement tronquées. Ne serait-ce que parce qu'il s'agit souvent d'évaluations, spécialement pour

8. Les Callouët sont une famille trégorroise déclarée noble de simple extraction par arrêt des commissaires de la réformation en date des 4 avril et 5 septembre 1669 et 18 août 1670 . Elle a porté les titres de baron de Trégomar et vicomte du Lou. Elle a produit un évêque de Tréguier, des avocats généraux à la chambre des comptes de Bretagne et de nombreux magistrats de Morlaix. Les Lanidy en constituent la branche aînée, qui disparaît avec Etienne René. Après son décès, ses biens passent en effet à son parent, Le Rouge de Guerdavid.

9. Arch. dép. du Finistère, 13 L 229.

10. Arch. dép. du Finistère, 1 Q 109, 1 Q 397, 1 Q 2039 et 1 Q 1209.

11. Pendant la Terreur, Callouët est interné et ses biens sont placés sous séquestre. Mais sous le Directoire, l'administration centrale du Finistère le raye de la liste des émigrés, Arch. dép. du Finistère, 1 Q 2626. 
la commission rarement mentionnée dans la baillée ou dans un autre document. Concernant la rente convenancière (loyer annuel dû par le domanier au foncier), nous avons fait un calcul à l'hectare. Cette manière de faire, apparemment rationnelle, se heurte toutefois à une réalité déjà rappelée : les parcelles ne se valent pas. On considérera donc les résultats obtenus comme des ordres de grandeur plutôt que comme des vérités. Ce préalable étant posé, on note un écart important entre les différents convenants. Ce n'est ni la taille, ni l'importance des terres fertiles, ni la complémentarité des parcelles qui détermine logiquement le montant de la rente convenancière. Ainsi les plus belles tenures (Kergariou, Feunteungoarec et Le Bodou), répondant le mieux aux trois conditions précitées, sont de très loin les moins imposées, alors que les plus petites (Serson, Penarstang Glaziou, Kergrech) le sont nettement plus. Comment l'expliquer? Il existe deux types de domaines congéables : les convenants proprement dits et les terres louées à domaine congéable. Parmi les convenants, il y a ceux qui sont exploités directement par l'édificier et ceux qui sont sous-loués. Ce sont les convenants exploités directement par le domanier, souvent depuis plusieurs générations, qui sont les moins pressurés. Ainsi Yves Le Foll, de Feunteungoarec, présente aux experts chargés d'évaluer le bien national une déclaration de 1654, ce qui signifie que, depuis cette date, le montant de la rente n'a pas bougé. Les moins favorisés sont les sous-domaniers, car à la contribution versée au foncier s'ajoute la ferme acquittée au domanier. Ce dernier, qui se décharge de la rente sur son locataire ${ }^{12}$, est peut-être moins tenté que le domanier exploitant directement son bien de résister au foncier.

Avec la commission, le foncier dispose d'une possibilité d'ajuster le prélèvement. Dans tous les cas elle est supérieure à une année de loyer. Elle n'atteint jamais deux années, excepté dans deux cas : Kergariou (x 3,2) et Lesconet Kergrist (x 11,2!). Mais il est vrai qu'il s'agit de beaux convenants au loyer particulièrement faible.

La clé de voûte ${ }^{13}$ du système convenancier est le congément, c'est-àdire la possibilité pour le foncier de congédier directement son domanier après évaluation et remboursement de ses droits, ou encore de transférer ce droit sur un tiers appelé subrogé. Les archives de la justice seigneuriale de Keralliou nous renseignent sur cette pratique à Botsorhel entre août 1782 et novembre $1790^{14}$. Pour ces huit années, outre le congément sujet de cette recherche, on trouve trace de sept expulsions. C'est peu, étant donné que le ressort de la justice de Keralliou s'étend à la presque totalité de la paroisse. On notera que les congéments ne se font jamais directement par le foncier et ne concernent en général qu'une partie des droits réparatoires. Cela signifie-t-il que l'opération se pratique le plus sou-

12. Et pas seulement de la rente si l'on en croit le cahier de doléances de Garlan, dans Roudaut, Fañch, Cahiers de doléances pour les états généraux de 1789, Quimper-Brest, 1989, plaquette de Garlan.

13. L'expression est de Philippe Jarnoux dans « Aux confins de la Basse-Bretagne : l'évolution du domaine congéable au XVIII ${ }^{\mathrm{e}}$ siècle ", Kreiz, 1995, p. 136.

14. Arch. dép. du Finistère, 17 B 153. 
vent à l'amiable entre paysans en vue de régler un héritage, d'épurer une dette ou d'assurer la rotation des parcelles les plus fertiles comme tendent à le démontrer les plus récentes recherches sur la question ${ }^{15}$ ? Sans trancher, on remarquera simplement que sauf dans un seul cas, jamais les parties n'ont réussi à se mettre d'accord sur le nom du troisième expert, de ce fait toujours nommé d'office. On constatera aussi qu'à deux exceptions près (dont une après la rédaction des cahiers de doléances et le début de la Révolution), le congément ne s'opère jamais au terme de la saint Michel, ce qui a certainement pour résultat de priver le domanier évincé des produits de sa moisson alors qu'il a déjà fumé, labouré et semé.

À quelle stratification sociale donne naissance une telle structure foncière? Les registres fiscaux ont à cet égard été d'un précieux secours ${ }^{16}$. Logiquement les métiers de la terre l'emportent. Si l'on exclut l'importante domesticité, ce qui frappe c'est la part des exploitants. Les journaliers représentent en effet un pourcentage infime. La catégorie la mieux représentée est celle des ménagers, qui se recrutent quasi exclusivement parmi les domaniers (encore qu'un nombre non négligeable d'entre eux, presque un sur cinq, soient étiquetés "ménagers-fermiers ", ce qui correspond aux sous-domaniers). La part de paysans "presque " propriétaires - du moins de leurs droits superficiaires - est donc très forte.

La contribution foncière de 1791 permet de se faire une idée de l'assise financière de cette paysannerie. Si les cotes les plus basses dominent, les cotes moyennes sont plutôt bien fournies et le sommet de la hiérarchie fiscale n'est pas spécialement maigre. Comme dans le Vannetais étudié par Tim Le Goff ${ }^{17}$, le domaine congéable accoucherait donc d'une société de petits et moyens paysans presque indépendants, avec à leur tête une élite accaparant les fonctions honorifiques ${ }^{18}$. François Madec et Allain Foll en font incontestablement partie. Le premier possède à Botsorhel les édifices et superfices de deux convenants, loue un troisième bien (il n'est pas précisé s'il s'agit d'un domaine congéable), emploie 4 domestiques, est capité à 20 livres en 1790 (17 pour lui et 3 pour ses domestiques), et acquitte une contribution foncière de 182 livres, 1 sou et 9 deniers en 1791 (la troisième cote de la paroisse, derrière celles de Callouët et de la Nation). Quant à

15. Voir en particulier GALLET, Jean, La Seigneurie bretonne (1410-1680). L'exemple du Vannetais, Paris, 1983; id., "Le congément des domaniers en Bretagne. Nouvelles perspectives de recherche ", Enquêtes et documents, 1980, p. 31-53; id., "Le congément des domaniers dans le Trégor au XVIII ${ }^{\mathrm{e}}$ siècle ", Mémoires de la Société d'Histoire et d'Archéologie de Bretagne, 1983, p. 143-160; id., " Le congément des domaniers en Cornouaille au XVIII ${ }^{\mathrm{e}}$ siècle ", Annales de Bretagne et des Pays de l'Ouest, 1983, p. 451-466; id., Seigneurs et paysans bretons du Moyen Âge à la Révolution, Rennes, 1992, p. 207-218; DupuY, Roger, « Domaine congéable et stratification sociale aux abords de Lorient à la fin de l'Ancien Régime ", dans ANTOINE, Annie (dir.), Campagnes de l'Ouest, stratigraphie et relations sociales dans l'histoire, Rennes, 1999, p. 355-360.

16. Arch. dép. du Finistère, 13 L 229 et 13 L 29.

17. LE Goff, Tim, Vannes et sa région. Ville et campagnes dans la France du XVIII siècle, Loudéac, 1989.

18. Kermoal, Christian, op. cit. 
Allain Foll, il est édificier de plusieurs domaines congéables, dont un pour lequel il est aussi le foncier (il achète le fonds de deux autres, devenus biens nationaux, en $1791^{19}$ ); il est capité à 20 livres (17 pour lui et 3 pour sa domesticité); il acquitte une contribution foncière de 92 livres, 10 sous et 4 deniers en 1791 ; il maîtrise parfaitement à la fois l'écrit et le français ${ }^{20}$. Notre homme effectue une belle carrière politique pendant la Révolution et l'Empire : signataire du cahier de doléances de la paroisse en $1789^{21}$, il est nommé électeur par l'assemblée primaire du canton de Guerlesquin en $1790^{22}$, entre dans l'administration du district de Morlaix quelques semaines après ${ }^{23}$, est élu officier municipal de sa commune en 1792 (en place jusqu'au Directoire), et occupe le poste de maire de l'an VIII à $1806^{24}$.

Reste maintenant à voir comment le système convenancier, largement dominant à Botsorhel donc, est perçu par ses usagers à l'aube de la Révolution. La préparation des États généraux permet de faire quelques remarques à ce sujet. Dans ce qui deviendra le Trégor finistérien, on sait, malgré le faible taux de conservation des cahiers de paroisse, que la question du domaine congéable a été jugée prioritaire par les ruraux. Une pétition, rédigée en janvier 1789 par les représentants des paroisses de Plouigneau, Lanéannou, Plourin, Plougasnou, Plougonven et Botsorhel, et destinée aux échevins de Morlaix en est la preuve ${ }^{25}$. Ce texte est doublement intéressant. D'abord parce qu'il démontre l'importance de la question convenancière pour les domaniers de la région. Ensuite parce qu'il prouve une nouvelle fois - s'il en était encore besoin suite aux travaux de Roger Dupuy $^{26}$ - que les ruraux n'ont pas reçu passivement les consignes venues des villes. Dans le cas présent, nous avons même une sorte de "cahier modèle " élaboré par les campagnes et proposé à une ville pour adhésion. En agissant ainsi, ces paroisses s'inscrivent dans un mouvement général qui touche l'ensemble de l'évêché de Tréguier au cours de l'hiver 1788-1789 27.

En introduction, les pétitionnaires (François Bivillon et Jean-François Foll pour Botsorhel) dénoncent "tous les abus qui se commetent depuis dix huit à vingt ans dans toute la basse Bretagne touchant aux domaines congéables ". Sur les quatre articles, le premier, de loin le plus long, développe cette idée et fait des propositions :

19. Arch. dép. du Finistère, 1 Q 272 (PV n ${ }^{\circ}$ 21). L'adjudication se fait en consortie. Parmi les cinq co-adjudicataires, il y a Guillaume Le Lay, député des sénéchaussées réunies de Morlaix et Lannion, avec lequel Allain Foll est probablement apparenté.

20. Sa lettre de soumission pour quatre biens nationaux (trois à Botsorhel et un à Plougonven) témoigne de cette bonne instruction, ibidem, 1 Q 257.

21. RoudAuT, Fañch, op. cit., plaquette de Botsorhel.

22. Arch. dép. du Finistère, 10 L 75.

23. Arch. dép. du Finistère, 10 L 80.

24. Arch. dép. du Finistère, $504 \mathrm{E}$ dépôt 1.

25. Arch. dép. du Finistère, 10 B 1.

26. Dupuy, Roger, De la Révolution à la chouannerie. Paysans en Bretagne 1788-1794, Paris, 1988, en particulier le chapitre I.

27. Kermoal, Christian, op. cit., II, p. 425-460. 


\begin{abstract}
"Les malheureux laboureurs après avoir travaillé long-tems pour mettre leurs terres en bon état, sont ordinairement obligés de les quitter ou du moins de payer une commission exorbitante, ou de sortir à la volonté du seigneur, chargés de femme, enfans \& betails, sans azile, ni ou se retirer. On discerne aisément ce mal; il serait trop long à expliquer. A ces égars supplions qu'il plaise à sa majesté arrêter le cours de ces congémens, que par trois motifs, $1^{\circ}$ à défaut des payemens des impots, tant royaux que seigneuriaux; $2^{\circ}$ Lorsque le seigneur désirera lui même, ou ses enfans, $3^{\circ}$ Lorsque les domainiers ne seront pas de bonnes moeurs; ou si mieux aime sa majesté, que tout bien à domaine congeable soit mis à titre de sens final, ou qu'au cas de congement que ceux qui n'auront pas [?] la moitié de leurs assurances la faculté donnée pour les congédier sera nulle, à commencer du $1^{\text {er }}$ janvier $1789 \&$ qu'aucun homme de justice ne pourra etre expert ni tiers d'office dans les congémens de campagne. "
\end{abstract}

C'est donc la suppression du domaine congéable ou du moins sa réforme qui est ici réclamée. L'argumentaire porte essentiellement sur la pratique du congément. Celui-ci ne devrait sanctionner que le mauvais domanier; il ne pourrait plus être aussi aisément utilisé par un rival cherchant à évincer son parent ou son voisin pour mettre la main sur son bien; enfin, il ne devrait plus être employé comme un instrument de " racket " permettant au foncier d'extorquer une commission plus forte pour prix du renouvellement de la baillée. À noter aussi l'idée d'un moratoire sur les congéments ainsi que le rejet des hommes de loi considérés comme trop partiaux du fait de leur implication dans le fonctionnement de l'institution seigneuriale.

Le cahier de doléances de Botsorhel, heureusement conservé, comporte neuf articles. L'article 5 porte plus spécialement sur le domaine congéable :

"Qu'il serait à désirer que le domaine congéable, qui a lieu en BasseBretagne, soit supprimé, en ce qu'il est très nuisible aux ménagers de la campagne; et, si on ne se portait pas à accorder cette suppression, qu'il ne fût au moins permis d'exercer les congéments que de Saint-Michel en SaintMichel, et non en aucun autre temps de l'année, que la commission soit fixée à une année de la redevance annuelle du seigneur, et enfin que le domanier ait à prendre sur sa tenue le bois nécessaire pour réparer ses édifices, faire ses charrettes, etc. ${ }^{28}$."

Là encore le désir d'une abolition de l'institution convenancière est mis en avant. En cas de refus, un compromis est cependant accepté. Ce dernier porte à nouveau sur la pratique du congément dont il s'agit de réduire l'arbitraire. Tout d'abord en fixant un calendrier que ne prévoit pas l'usement de Tréguier. Ensuite en bloquant le montant de la commission de façon à ce que l'expulsion ne puisse plus être utilisée comme moyen de chantage pour l'aggraver. Le cahier pose aussi la question des bois. Les usements accordent en effet au foncier tous les arbres de la surface, sauf les fruitiers. Quant à ceux des fossés, les troncs lui appartiennent. Le domanier ne peut donc abattre d'arbres sans autorisation. Et s'il plante, le fruit de son travail

28. RoudAuT, Fañch, op. cit. 
revient au foncier. Le résultat d'une telle situation est la pénurie de bois et, conséquemment, sa cherté. Pénurie d'autant plus accentuée que les colons ne se sentent nullement encourager à boiser.

En remettant ainsi en cause le domaine congéable, la paroisse de Botsorhel et celles du pays morlaisien ne sont point isolées : c'est toute la Bretagne acconvenancée qui en fait le nœud de la question agraire ${ }^{29}$. Le fait divers qui agite Botsorhel et les campagnes voisines quelques mois plus tard le confirme.

\section{L'affaire du congément}

L'affaire commence le 4 août 1790 par une demande en congément déposée en la juridiction de Keralliou. Une suite favorable lui est donnée un mois plus tard par le juge seigneurial, Ladvenant de Kerisac; l'expulsion est fixée à la saint Michel ${ }^{30}$. Auparavant, le seigneur du lieu et propriétaire du fonds, Callouët de Lanidy, avait par une baillée de congément autorisé Maurice Hemeury, Jacques Le Balch et Jean Le Morin à congédier Laurent Le Guilloux, Laurent Le Lay et leurs consorts du convenant du Rhun. Dans cette affaire, demandeurs et défendeurs ne sont pas des inconnus, ils sont même très étroitement liés par les liens de la consortie, de la famille et du voisinage. Quelques mois auparavant, Laurent Le Guilloux avait réclamé et obtenu en sa faveur le retrait lignager de la portion du convenant appartenant à Laurent Le Lay et à son épouse ${ }^{31}$. Faut-il voir dans le congément une réponse à cette affaire?

L'opération de prisage, mesurage et estimation a lieu le 15 octobre. Les trois experts sont : Jean-François Le Scornet pour les demandeurs, Ambroise Louis Troussel pour les défendeurs, Jean-François Prigent, tiers nommé d'office faute aux parties de s'être mises d'accord à l'amiable. Parvenus au Rhun les trois experts et les demandeurs trouvent les époux Le Lay et Guillou ainsi que Jean Thépaut, les trois hommes armés d'une faucille. Vivement et bientôt avec colère, ces derniers refusent d'effectuer la montrée de leurs droits superficiaires et chassent les intrus. Ils se justifient de la manière suivante :

" le domaine congéable est aboly par un decret de l'assemblée nationale, ainsy que les justices seigneuriales, [ils] tiennent ce fait du maire \& des officiers municipaux de Botsorhel qui leur ont recommandé de renvoyer les priseurs qui prétendroient arpenter et priser leurs droits, [ces derniers sont] sans qualité pour faire des prisages, et [ils] s'oppos[ent] formelement a toute espece de mésurage et prisée dans le dit convenant Le Rhun, [ils] ne [leur] fer[ont] aucune montrée et [ils] defi[ent] qui que ce soit d'en faire et les [experts] d'opérer et que [s'ils] ne [se] retir[ent] proptement et sans autre

29. LE BlOAS, Alain, « La question du domaine congéable dans l'actuel Finistère d'après les cahiers de doléances ", Annales Historiques de la Révolution Française, n 331 , janviermars 2003, p. 1-27.

30. Arch. dép. du Finistère, 17 B 153.

31. Arch. dép. du Finistère, 17 B 153. 
formalité, ils saur[ont] bien [les] y forcer, qu'au surplus, s'il avoit été permis de faire des congéments, ils se fussent volontiers rapporté à [leur] prisée ${ }^{32}$. "

À l'instar de bien des paysans bas-bretons, ceux de Botsorhel, dans leur hâte de voir concrétiser les attentes exprimées au printemps 1789, interprètent le décret du 15 mars 1790 promettant de légiférer sur le domaine congéable comme suspendant ce dernier. Devant cette résistance, experts et congédiants se retirent, poursuivis par Le Lay et Thépault toujours aussi menaçants.

Informé de l'incident, le directoire du district de Morlaix décide d'écrire à la municipalité de Botsorhel qu'elle convoque au chef-lieu dans le but de l'admonester. Elle fait de même auprès de Allain Foll afin qu'il intervienne auprès des édiles et les remette dans le droit chemin. Quelques jours après, le maire accompagné d'un officier municipal se rend à la convocation du directoire. Mais "fondez sur un écrit dont ils [sont] saisis "33", ils refusent d'en démordre. Quant au juge seigneurial de Keralliou, une nouvelle fois sollicité par les demandeurs, il réitère son premier jugement.

Un mois plus tard, le 17 novembre, une nouvelle tentative de prisage a donc lieu. Cette fois-ci sous la protection de deux cavaliers dépêchés par la maréchaussée de Morlaix. Les experts ont changé : Jean Le Noan pour les demandeurs, Pierre Marie Capitaine pour les défendeurs, et Jean François Troadec, nommé d'office. À proximité du Rhun, la petite troupe rencontre quatre hommes postés qui la suivent. Sur place, le comité d'accueil s'étoffe. Le Lay et Le Guilloux refusent à nouveau de procéder à la montrée. Ils exhibent un papier - sans doute le même que celui dont s'étaient prévalus les élus municipaux devant les administrateurs - signé Le Coursonnois ${ }^{34}$, procureur de Poullaouën, rédacteur d'une pétition réclamant la suppression du domaine congéable à l'occasion de la première assemblée électorale du Finistère en juin $1790^{35}$, où il est écrit : "Les juridictions etant supprimées la justice de Kerallio [est] sans qualité et [...] les défendeurs [doivent] s'opposer à tous prisage de leurs droits convenanciers ${ }^{36}$. " Les épouses Le Lay et Le Guilloux entrent alors en scène. L'une pénètre dans un champ et en fait sortir onze personnes composant la municipalité, le maire Jean Le Foll en tête. L'autre conduit une petite troupe dont certains membres sont armés de bâtons. Le maire affirme que les experts ne peuvent agir sans l'avis des élus qui ajoutent : «Les défendeurs [sont] les maîtres de leurs droits et [...] ils [ne sont] venu audit bien que pour [les] empecher de faire du mal ${ }^{37}$. " La foule encercle alors les experts et la maréchaussée qui décident de battre retraite. À ce moment les menaces pleuvent et Le Balch est molesté. La municipalité

32. Arch. dép. du Finistère, 17 B 153.

33. Arch. dép. du Finistère, 26 L 12.

34. Simon Marie Le Coursonnois : officier des mines; procureur de la commune de Poullaouën en 1790; électeur titulaire du canton de Carhaix en 1790, suppléant en 1791; régulièrement nommé au bureau provisoire ou définitif des assemblées primaires et électorales.

35. Arch. nat., D/XIV/3.

36. Arch. dép. du Finistère, 17 B 153.

37. Arch. dép. du Finistère, 17 B 153. 
rédige ensuite une adresse à l'Assemblée nationale dans laquelle Callouët de Lanidy est peint " sous les traits les plus défavorables, comme un homme acharné contre les cultivateurs ${ }^{38}$ ". Informée de cette nouvelle rébellion, l'administration morlaisienne décide de transmettre l'affaire au département.

Si Maurice Hemeury abandonne sa démarche de congément (sous la pression de la communauté? suite à une conciliation?), Jacques Le Balch, lui, ne désarme pas et porte plainte auprès du tout nouveau tribunal du district de Morlaix contre le seul Le Guilloux. Les juges lui donnent raison en ordonnant l'expulsion du défendeur et en menaçant de recourir à la force publique en cas de nouvelle rébellion, en condamnant Le Guilloux à verser 120 livres de dommages et intérêts au plaignant et 12 livres d'amende au tribunal; ils placent aussi Le Balch sous la sauvegarde de la justice en prévision d'une possible vengeance ${ }^{39}$.

Les mêmes experts que ceux nommés pour la seconde tentative de montrée, accompagnés cette fois-ci d'un huissier, reviennent au Rhun le 21 juin. Le front " anticongément " qui s'était précédemment constitué semble s'être rompu : les consorts Le Lay, la municipalité et les habitants de Botsorhel sont en effet absents. Anne Cloarec, épouse de Laurent Le Guilloux, est seule. Elle affirme pourtant

" que son mari avoit parti pour prevenir le sieur Coursonnois de se trouver au dit convenant le run lequel Coursonnois devoit etre rendu depuis hier au Guerslesquin avec differents particuliers de poullaouen et qu'ils auroient en la vie de l'un ou de l'autre de nous [nous = les experts] ainsi que celle dudit le Balch demandeur ${ }^{40}$."

Mais personne ne vient. Anne Cloarec demande alors à un de ses enfants d'ameuter le voisinage. Là encore, aucun écho. Lassitude et découragement face à l'intransigeance de l'administration et de la justice, alors que la Constituante s'est ralliée au point de vue des partisans du maintien du domaine congéable? Sentiment d'avoir obtenu en partie gain de cause en limitant le congément au seul Le Guilloux? Agacement devant l'irascibilité du couple et en particulier de l'épouse? Saisie par la colère, cette dernière s'empare de différents objets avec lesquels elle tente de frapper les hommes de loi. En vain, la prisée se fait et le congément s'opère.

38. Arch. dép. du Finistère, 26 L 12. Le texte de cette adresse n'a pas été retrouvé, le registre de la municipalité de Botsorhel pour l'époque des faits ayant hélas disparu.

39. Arch. dép. du Finistère, 73 L 7. Quelle est l'attitude des nouvelles juridictions, dont les domaniers attendaient plus d'impartialité, lors des demandes de congément? Le tribunal de Rostrenen avait refusé à trois reprises de juger un congément sous prétexte que l'Assemblée nationale pourrait bientôt abolir cette pratique (dans Léon Dubreuil, op. cit., I, p. 389-390). Pour le premier semestre de l'année 1791 (Arch. dép. du Finistère, 73 L 6, 7 et 15), nous avons constaté que le tribunal de Morlaix, lui, donnait toujours raison aux fonciers et aux subrogés, qu'il s'agisse de congéments ou d'arrérage de rente foncière. Mais il est aussi à noter que les demandes de congément pour l'ensemble du district sont devenues rarissimes (6 avec celle du Rhun), alors que, si l'on en croit les différents mémoires et pétitions, les expulsions s'étaient multipliées entre le déclenchement de la Révolution et l'extinction des justices seigneuriales. La mobilisation convenancière n'est sans doute pas étrangère à ce résultat.

40. Arch. dép. du Finistère, 73 L 69. 
Voilà donc l'affaire telle que les papiers de la justice de Keralliou, ceux du tribunal et du directoire du district de Morlaix la révèlent. À première vue rien de très intéressant : un simple fait divers, probablement motivé par une mésentente familiale. Les protagonistes eux-mêmes semblent insignifiants.

En 1790, Laurent Le Lay tout comme Maurice Hemeury sont capités à une livre, Jacques Le Balch et son beau-frère à 2 livres; aucun d'entre eux n'emploie de domestiques ${ }^{41}$. Pour la même année, concernant les vingtièmes et les fouages cette fois-ci, Laurent Le Lay et ses consorts sont imposés pour la somme de 7 livres et 2 sous, Jacques le Balch pour celle de 26 livres et 7 sous $^{42}$. À la contribution foncière de 1791, Laurent Le Lay est imposé à 21 livres, 9 sous et 9 deniers, Jacques Le Balch à 23 livres et 13 deniers $^{43}$. À la contribution mobilière, Maurice Hemeury est imposé à 2 livres et 10 sous, Jacques Le Balch et son beau-frère à 5 livres et 15 sous, Laurent Le Lay à 3 livres et 5 sous, Jean Le Morin à 2 livres et 5 sous ${ }^{44}$.

Si Jacques Le Balch, manifestement le plus aisé, est à la tête de deux convenants, son compère demandeur, Maurice Hemeury, n'est même pas propriétaire : il cultive un petit domaine congéable peu boisé de sept journaux et trente-trois cordes, comprenant aussi une maison, une crèche, un puits et un four, le tout estimé à 535 francs lors de l'expertise menée en l'an VII; Julienne Duparc, la domanière en titre, le lui sous-loue ${ }^{45}$. Quant à la partie du Rhun appartenant à Laurent Le Guilloux, un huitième du tout selon ce dernier, elle est constituée d'une maison, d'une aire sans valeur et de quelques cordes de terre, le tout estimé par les experts à 196 livres, 18 sous et 9 deniers ${ }^{46}$.

Aucun des protagonistes directs de l'affaire ne peut être considéré comme un notable. Si Maurice Hemeury figure peut-être parmi les délibérants du corps politique de la paroisse au vu des signataires du cahier de doléances de $1789^{47}$, si Guillaume Le Balch, Jean Le Morin et Laurent Le Lay apparaissent dans une liste des citoyens actifs composant la garde nationale en $1792^{48}$, nul ne semble appartenir la municipalité élue en $1790^{49}$. Aucun ne figure dans celle de l'an III ${ }^{50}$, ni parmi les quatre assesseurs de Botsorhel du juge de paix du canton de Guerlesquin élus en décembre

41. Arch. dép. du Finistère, 13 L 229.

42. Arch. dép. du Finistère, 13 L 12.

43. Arch. dép. du Finistère, 13 L 29.

44. Arch. dép. du Finistère, 13 L 29.

45. Arch. dép. du Finistère, 1 Q 397 (propriétaire du fonds : Mouillemuse, émigré).

46. Arch. dép. du Finistère, 73 L 69.

47. Fañch RoudAut, op. cit. Le registre du général de paroisse, qui aurait permis de le vérifier, a disparu.

48. Arch. dép. du Finistère, 26 L 118.

49. Arch. dép. du Finistère, $521 \mathrm{E}$ dépôt art. 2. À cette occasion, la municipalité de Botsorhel était représentée par six de ses membres (le maire, le procureur, 4 officiers municipaux).

50. Arch. dép. du Finistère, 26 L 38. 
$1790^{51}$, et encore moins parmi les électeurs du second degré nommés en $1789,1790,1791$ et $1792^{52}$. En somme, pas un seul personnage impliqué directement dans le congément ne peut se prévaloir d'une quelconque appartenance à la sanior pars de la paroisse.

C'est pourtant cette banalité, pour ne pas dire cette médiocrité des acteurs, qui donne toute sa force à l'affaire. En effet, la division qui affecte à cette occasion la communauté de Botsorhel ne peut être interprétée comme le résultat d'une lutte entre deux partis adverses. La mobilisation, sans faille à ses débuts, d'une partie des habitants, édiles en tête, autour des congédiés est bien dirigée contre la seule pratique du congément, et, par extension, contre le domaine congéable.

Cette donnée dément la thèse d'une institution instrument de régulation sociale et familiale aux vertus quasi magiques, acceptée et utilisée par tous. Les cahiers de doléances avaient, dans un premier temps, permis à ce propos de libérer une parole jusque là comprimée. La Révolution, elle, offre enfin aux domaniers la possibilité de résister ouvertement. Sous l'Ancien Régime, combien d'expulsés avaient dû en réalité se soumettre à la montrée les larmes aux yeux et les poings serrés parce qu'ils ne pouvaient faire autrement? Les archives, dans leur sécheresse toute juridique, sont muettes à ce propos. Pourtant nul doute qu'il y en eut beaucoup.

Elle met aussi en cause une approche purement sérielle de la pratique du congément, test qui permettrait de supputer une réception positive ou négative de l'institution convenancière parmi ses usagers. Ce n'est pas la quantité de congéments qui rend le domaine congéable impopulaire, c'est le congément lui-même. Et le caractère exceptionnel qu'il a acquis au $\mathrm{XVIII}^{\mathrm{e}}$ siècle le rend d'autant plus inadmissible ${ }^{53}$. Avec les restrictions mises à l'usage des bois, il rappelle en effet à cette démocratie de petits et moyens paysans, souvent présents sur leurs convenants depuis plusieurs générations, que leurs droits convenanciers ne leur appartiennent pas en réalité et peuvent un jour leur être brutalement enlevés.

L'événement a bien évidemment eu un écho dans les autres paroisses. Son caractère séditieux conjugué à cet effet amplificateur, alors que la mobilisation convenancière bat son plein, a contribué à une prise de position des administrateurs du district de Morlaix et du département du Finistère, jusque là étrangement muets sur la question du domaine congéable.

\section{Les répercussions de l'affaire}

L'affaire de Botsorhel survient alors que la campagne abolitionniste est à son apogée. La période précédant les États Généraux en a marqué le démarrage. La fédération de Pontivy, durant l'hiver 1790, l'a relancée et

51. Arch. dép. du Finistère, 73 L 61.

52. Arch. dép. du Finistère, 10 L 75 et 10 L 79; Fañch Roudaut, op. cit.

53. Philippe JARNOUX, art. cité, p. 136-139. 
l'a structurée. Puis les premières assemblées primaires et électorales, entre mai et juillet 1790, ont été utilisées comme caisses de résonance pour leurs revendications par les domaniers et leurs alliés ${ }^{54}$. Délibérations, pétitions et mémoires se multiplient alors pendant le second semestre de l'année 1790, avec une accélération lors du dernier trimestre provoquée par les nouvelles consultations électorales, en particulier la mise en place des premières justices de paix, et l'annonce de la réunion du conseil général du Finistère. Ceci au moment même où, à la Constituante, les députés Le Lay ${ }^{55}$ et Baudoin ${ }^{56}$ élaborent des projets de décrets antagonistes mais ayant tout de même en commun d'être réformistes, alors que la paysannerie de Basse-Bretagne s'est depuis les cahiers de doléances largement radicalisée en évoluant vers des positions désormais majoritairement abolitionnistes.

De tous les districts finistériens, celui de Morlaix est certainement le plus actif, particulièrement dans sa partie trégorroise et dans ses marges léonardes. À la fin de l'année 1789 ou au début de 1790, les paroisses trégorroises de Plougonven, Plourin, Plouégat-Moysan, Garlan, Plouigneau, Plougasnou et Lannéanou, ainsi que celle léonarde de Taulé, se fédèrent pour délibérer; par l'intermédiaire de Le Lay, leurs représentants s'adressent au comité féodal qu'ils pressent d'agir ${ }^{57}$. En réponse à cette offensive domanière, 109 propriétaires fonciers habitant Morlaix rédigent à leur tour des pétitions auprès de l'Assemblée nationale; ils reçoivent le soutien de la municipalité morlaisienne, qui de plus se désolidarise de l'assemblée de Pontivy auprès de laquelle elle avait pourtant député ${ }^{58}$. Lors de la formation du département en juin, les électeurs du district de Morlaix sont avec ceux du district de Quimper les plus prompts à signer l'Adresse des cultivateurs de Léon et Cornouaille ${ }^{59}$. Au cours du dernier trimestre de l'année 1790 , l'activité pétitionnaire reprend de plus belle : Plouézoc'h ${ }^{60}, \operatorname{Garlan}^{61}$,

54. LE BLOAS, Alain, «Voter et élire en Basse-Bretagne au début de la Révolution : les toutes premières assemblées électorales dans le Finistère ", Bulletin de la Société archéologique du Finistère, 2003, p. 347-371

55. Guillaume Le Lay : né en 1742; domanier à Lannéanou; élu député des sénéchaussées réunies de Morlaix et de Lannion pour les États Généraux; administrateur du district de Morlaix en 1792.

56. Jean Marie Baudoin de Maisonblanche : né en 1742; avocat à Lannion; auteur des Institutions convenancières en 1776; élu député de Lannion aux États de Bretagne, puis membre de la commission intermédiaire pour l'évêché de Tréguier; élu député des sénéchaussées réunies de Morlaix et de Lannion pour les États Généraux; maire de Lannion de novembre 1791 à novembre 1792; préside le directoire de l'administration centrale des Côtesdu-Nord de l'an IV à l'an VIII; conseiller de préfecture sous le consulat; décède en 1812.

57. Léon DubreulL, op. cit, p. 370 sqq. La lettre ne mentionne aucune date, mais elle a certainement été rédigée juste avant l'élection des premières municipalités.

58. SAGnac, Philippe et CARON, Pierre, op. cit., p. 126-136.

59. Arch. nat., D/XIV/3.

60. Arch. dép. du Finistère, 10 L 227. La municipalité et le canton de Plouézoc'h émettent trois pétitions coup sur coup. Là encore elles ne sont pas datées, mais les informations qu'elles recèlent permettent d'en situer à coup sûr la rédaction à l'automne 1790.

61. Arch. dép. du Finistère, 67 G 4. 
Ploujean ${ }^{62}$, Plounéour Ménez ${ }^{63}$ et Plouigneau ${ }^{64}$ se mettent ainsi en valeur. Quant aux municipalités du canton de Guerlesquin (Guerlesquin, Botsorhel, Plouégat-Moysan et Le Ponthou), assemblées le 27 novembre en vue de préparer la prochaine assemblée primaire qui doit permettre la mise en place de la justice de paix, elles délibèrent aussi sur le sujet :

"L'assemblée après avoir ouï et pris lecture d'un projet de decret sur les domaines congeables proposé par M. lelay deputé à l'assemblée nationale \& ouï M. Guiot p[rocureu]r de la Commune [de Plouégat-Moysan] en adherant aux demandes et petitions prises dans l'assemblée générale de pontivy et par le corps electoral de morbian a arrêté de demander la supression \& abolition totale de la tenure à domaine congeable ${ }^{65}$."

Le texte n'évoque pas le conflit qui vient de secouer une des communes du canton et à laquelle plusieurs des délibérants ont pourtant activement participé, mais nul doute qu'elle a été évoquée et a contribué à cette prise de position. Dans son argumentaire, la municipalité de Garlan, elle, évoque les « insurrections auxqu'elles les remboursemens commencent à donner lieu ${ }^{66}$ ". Ne faut-il pas voir là une allusion implicite au congément sulfureux de Botsorhel?

La preuve que l'information circule effectivement largement à ce propos, nous la trouvons dans cette délibération de la commune costarmoricaine de Loc-Envel (district de Guingamp) :

"Le tocsin sonne dans une de nos paroisses. Trois fois les priseurs ont descendu pour apprécier un lieu, accompagnés de cavaliers de maréchaussée, d'huissiers et assistants. Trois fois ils ont été repoussés par les courageux habitants de cette paroisse ${ }^{67}$."

Même les paroisses rurales du canton cornouaillais de Carhaix évoquent le cas lors de l'élection de leur juge de paix :

"La fureur des congéments est si grande, que les parents les plus proches, sans égard aux liens du sang, et jusqu'aux frères et soeurs s'entrecongédient; à ce moment un pareil congément s'exerce en Tréguier, paroisse

62. Arch. dép. du Finistère, 26 L 71.

63. Arch. municipales de Plounéour Ménez. La délibération de la municipalité de Plounéour est occasionnée par une lettre de Le Lay transmise au maire par les officiers municipaux de Pleyber-Christ (les deux communes constituent le canton de Pleyber-Christ).

64. Nous n'avons pas retrouvé la pétition de Plouigneau, mais les archives du département nous apprennent que la municipalité a pétitionné contre le domaine congéable le 5 décembre et fait parvenir le texte au directoire du Finistère.

65. Arch. dép. du Finistère, $521 \mathrm{E}$ dépôt art. 2. L'assemblée générale de Pontivy correspond bien sûr à la deuxième fédération tenue en février 1790. Quant à la pétition du corps électoral du Morbihan, destinée à l'Assemblée nationale, elle a été rédigée en juin 1790 à l'occasion de la mise en place de la première administration départementale.

66. Arch. dép. du Finistère, 67 G 4.

67. Léon DuBREulL, op. cit., I, p. 327. Dans une note en bas de page, l'auteur voit dans ces lignes une allusion à un événement similaire survenu à Loguivy-Plougras. Il se trompe vu que la délibération date du 13 décembre 1790, alors que le congément de Loguivy-Plougras, lui, débute en mars 1791. Même si les faits sont rapportés avec exagération par les édiles de Loc-Envel, c'est donc bien au conflit survenu à Botsorhel qu'il est fait allusion. 
de Botsorhel. Le ci-devant seigneur de Lannidy (Calloët) a donné une baillée à Maurice Hemery pour congédier ses frères et sa sœur sans égard à la règle que la cour est saisie, et au décret du 15 mars qui réserve de statuer par une loi expresse sur le domaine congéable ${ }^{68}$."

Il est vrai que le rédacteur de la pétition n'est autre que le fameux Le Coursonnois, considéré comme leur maître à penser par les édiles de Botsorhel. L'affaire de congément survenue au printemps 1791 dans la proche paroisse costarmoricaine de Loguivy-Plougras et évoquée par Léon Dubreuil $^{69}$ ressemble fortement à celle née quelques mois plus tôt à Botsorhel, qui, à cette époque, alors que le tribunal de Morlaix n'a toujours pas tranché, fait encore figure de victoire pour les domaniers. Il n'est pas déraisonnable de penser que Botsorhel a par son exemple contaminé Loguivy-Plougras.

Aussitôt élu, le conseil général du Morbihan réclame à la Constituante la suppression du système convenancier ${ }^{70}$. Quelques semaines après, sans prendre partie, le directoire demande à l'Assemblée nationale de se prononcer rapidement sur la question ${ }^{71}$. Dans ce département, le district de Pontivy se montre particulièrement militant ${ }^{72}$. Entraînée par des initiatives des districts de Lannion, de Rostrenen, et, surtout, de Guingamp, d'où part la fameuse pétition de Huchet et Labat, transformée en véritable référendum par les ruraux de la partie acconvenancée du département, l'administration départementale des Côtes-du-Nord fait de même malgré son désir de ne pas se compromettre ${ }^{73}$.

L'accélération de l'agitation convenancière dans le dernier trimestre de l'année 1790 précipite la prise de position des administrations supérieures du Finistère qui, contrairement à leurs homologues des deux autres départements bretons, sont dans l'ensemble restées muettes sur la question du domaine congéable. Seuls deux districts finistériens se sont alors prononcés. Le directoire de Quimper, qui se fait l'interprète des électeurs, réclame au conseil général qui doit se réunir de souscrire à une pétition réclamant l'abolition ou du moins la réforme du régime convenancier ${ }^{74}$. Par conviction et parce que le district contient de nombreux convenants nationaux qu'il faut mettre en vente, celui de Quimperlé, en contradiction totale avec le vou des électeurs du district ${ }^{75}$, renouvelé et radicalisé par le conseil d'administration ${ }^{76}$, empêche la diffusion dans les campagnes des lettres de Le Lay au motif suivant " nous sommes davis de [...] conserver [les domaines

68. SAGnac, Philippe et CARON, Pierre, op. cit., p. 492.

69. Dubreuil, Léon, op. cit., I, p. 391-404; id., " La lutte économique... ", art. cité.

70. Dubreull, Léon, Les vicissitudes..., op. cit., p. 209-211.

71. Sagnac, Philippe et Caron, Pierre, op. cit., p. 477-478; Kermoal, Christian, op. cit., II, p. 487-488, a cartographié la réception de cette pétition dans les campagnes trégorroises.

72. Dubreuil, Léon, op. cit., I, p. 212-219.

73. SAGnac, Philippe et CARON, Pierre, op. cit., p. 470-476; DuBreull, Léon, op. cit., p. 208-209.

74. DuBREUIL, Léon, op. cit., p. 219-225.

75. Arch. dép. du Finistère, 29 L 1.

76. Arch. dép. du Finistère, 29 L 2. 
congéables] et dans toutes les occasions nous avons exprimé notre vœux pour leur maintien ${ }^{77}$ ". Et fait officiellement connaître cette position après en avoir délibéré, demandant qu'elle soit transmise à l'Assemblée nationale :

"Nous ne pouvons vous le dissimuler et vous le savez mieux que nous que l'interet de la nation est essentiellement lié à celui des propriétaires fonciers, surtout pour nos ventes actuelles qui en souffriroient beaucoup ${ }^{78}$."

L'affaire de Botsorhel oblige le district de Morlaix à se déclarer à son tour. Ce dernier compte six administrateurs issus de la paysannerie, soit la moitié de son effectif. Parmi eux, Allain Foll, Jean-Marie Clech, Jacques Quéinnec et Jacques-Laurent Le Lamer ${ }^{79}$ ont été identifiés comme domaniers. Mais on l'a vu avec le district de Quimperlé, l'équipe dirigeante n'est pas forcément au diapason de son conseil d'administration. Ainsi Le Dissez ${ }^{80}$, procureursyndic du district, est-il l'auteur d'une plaidoirie en faveur de l'institution convenancière qu'il adresse au comité féodal en mars 1790 : les Réflexions d'un laboureur armoricain sur le domaine congéable de Tréguier ${ }^{81}$. Quant à Mathieu Beaumont ${ }^{82}$, vice-président du directoire mais aussi receveur de la

77. Arch. dép. du Finistère, 29 L 3. Cette position du directoire et du procureur syndic, déjà très perceptible lors des deux pétitions précitées, apparaît encore plus nettement dans une lettre datée du 4 septembre où l'administration quimperloise soutient avec succès auprès du directoire du Finistère l'idée d'évaluer les convenants nationaux au denier 22 plutôt qu'au denier 20 comme le prévoit la loi sur les biens nationaux.

78. Arch. dép. du Finistère, 21 L 227. La délibération, en date du 20 décembre, est imprimée à Paris sur 16 pages (nous ne l'avons pas retrouvée aux archives départementales). Le Quinio, qui y est attaqué, y répond dans Dernières réflexions sur le domaine congéable, Paris, 1791, $44 \mathrm{p}$.

79. Allain Foll : cf. supra. Jean-Marie Clech : domanier à Plougasnou; député de sa paroisse, puis de la sénéchaussée de Morlaix en 1789; nommé électeur de son canton (il défend le principe de l'indemnisation des électeurs lors de la première assemblée électorale du département à Quimper), et administrateur du district de Morlaix en 1790; à nouveau électeur en 1792; sans doute membre de la municipalité cantonale de Plouézoc'h en l'an IV. Jacques Quéinnec : né en 1755; domanier et marchand de toile à Plounéour-Ménez; député de sa paroisse en 1789 (il manque de peu l'élection à Versailles lors de l'assemblée électorale de la sénéchaussée de Lesneven); élu procureur de sa commune en 1790, puis électeur de son canton, et enfin administrateur du district de Morlaix (nommé administrateur du département quelques jours auparavant, il avait refusé la fonction); l'assemblée électorale du département le nomme député à la Convention en 1792 (il est décrété d'arrestation en 1793 pour avoir protesté contre la violation de la représentation nationale, il retrouve son siège après Thermidor); en 1795, l'assemblée électorale de France le choisit pour siéger aux CinqCents où, de concert avec Bohan, il tente d'empêcher le rétablissement du domaine congéable; il quitte la représentation nationale en 1798 et rentre alors dans la vie privée (il figure toutefois parmi les conseillers d'arrondissement en 1801); il s'installe à Guiclan où il meurt en 1817. Jacques-Laurent Le Lamer : domanier à Taulé; électeur en 1790, il devient administrateur du district de Morlaix et entre au directoire.

80. François-Marie Le Dissez-Kerbabu : né en 1726; avocat à Morlaix; subdélégué de l'intendant; élu administrateur puis procureur syndic en 1790, quitte cette fonction en 1791 ; juge au tribunal de district en 1794; membre de la municipalité après Thermidor; membre du conseil d'arrondissement en 1801.

81. DuBREUIL, Léon, op. cit., I, p. 167-172; KeRMOAL, Christian, op. cit., II, p. 486.

82. Mathieu Beaumont : né en 1723; banquier et négociant à Morlaix; député à l'assemblée du Tiers à Rennes en 1789; élu administrateur en 1790, il entre au directoire (très 
duchesse de Lévi, il est présenté par les habitants de Plouézoc'h dans une pétition anti-domaine congéable comme « l'agent qui [les] accable le plus, tandis qu'en sa qualité d'administrateur de district, il devroit etre plus humain $^{83}$ ". Le Lamer, lui aussi directeur, bien que domanier n'en est pas moins demandeur en congément contre Guillaume Le Roux, édificier à Taulé $^{84}$. Tout comme Jean-Marie Clech, simple administrateur, contre son frère François pour le quart d'un convenant à Plougasnou ${ }^{85}$, ainsi que son collègue Allain Foll - par ailleurs signataire de l'Adresse des cultivateurs de Léon et Cornouaille où les congéments sont vilipendés - contre François Callarec et consorts, domaniers à Botsorhel.

Le district doit se prononcer sur le fond de l'affaire - la question du domaine congéable - mais aussi sur sa forme, à savoir la désobéissance aux lois des habitants de Botsorhel et surtout de la municipalité. Concernant ce dernier point, comme nous l'avons vu, l'attitude du directoire a été ferme dès le départ. Le comportement de la municipalité lui paraît particulièrement blâmable : elle "s'est grievement compromise par la résistance conseillée, par les sentiments qu'elle a manifester au lieu de maintenir la paix et le bon ordre, enfin par son obstination a ne pas defferer aux sages avis que nous leur avons donné ${ }^{86}$ ". Concernant le premier point, il se contente de réclamer l'accélération du travail des législateurs, sans donner son opinion sur la direction qu'ils devraient suivre, à savoir la réforme - légère ou sévère - ou l'abolition :

" Nous pensons, MM., qu'il serait bien intéressant pour la tranquilité des campagnes de solliciter de l'assemblée nationale une prompte décision sur le domaine congéable afin de prevenir toutes révoltes ultérieures qui ne manqueront pas de se propager indubitablement si elles demeurent impunies et de causer peut-être les plus grands malheurs ${ }^{87}$."

Le conseil général du département, assemblé pour tenir sa session annuelle, nomme le 5 novembre 1790 une commission constituée de Veller, Créachquérault et Arnoult ${ }^{88}$; elle est chargée de réfléchir à la manière d'esti-

rapidement il manifeste son désaccord avec la politique religieuse de la Constituante et son application par l'administration départementale en présentant sa démission qui lui est refusée); désigné comme suspect, il est arrêté par la municipalité morlaisienne en septembre 1792 ; il est à nouveau emprisonné pendant la Terreur; siège au conseil d'arrondissement en 1801; décède en 1811.

83. Arch. dép. du Finistère, 10 L 227.

84. Arch. dép. du Finistère, 73 L 6, 73 L 15 et 73 L 69.

85. Arch. dép. du Finistère, 73 L 6, 73 L 15 et 73 L 69.

86. Arch. dép. du Finistère, 26 L 12. Lettre au directoire du Finistère, le 26 novembre 1790. 87. Arch. dép. du Finistère, 26 L 12. Lettre au directoire du Finistère, le 26 novembre 1790.

88. Louis Nicolas Veller de Kersalaun : né en 1734; avocat à Carhaix; maire de la ville sous l'Ancien Régime et député aux États de Bretagne en 1776 et 1788, membre de la commission diocésaine; élu maire par les Carhaisiens en 1790; après avoir été nommé électeur, il devient administrateur du département et entre au directoire la même année; administrateur du district de Quimper en 1792; compromis dans l'aventure fédéraliste, il est emprisonné; libéré après Thermidor, il retrouve la fonction de maire de Carhaix; membre de la municipalité cantonale sous le Directoire; juge de paix en 1816; il meurt 
mer les bois placés sur les convenants nationaux en vue d'éclairer les municipalités chargées des ventes ${ }^{89}$. Les pétitions dirigées contre le domaine congéable se multipliant, cette commission, d'abord purement technique, est à la demande de Capitaine ${ }^{90}$, le procureur général syndic, chargée de réfléchir à la question plus vaste du domaine congéable en vue de dresser un projet d'arrêté exprimant « le vœu commun des habitants du Département et qui soit ensuite soumis aux Lumières et à la sanction de l'Assemblée nationale ${ }^{91}$ ". Sont élus pour la composer : Arnoult, Cosson, Carquet et Veller, le dernier cité se comportant en véritable cheville ouvrière de l'équipe ${ }^{92}$. Il est à noter que les deux membres de l'administration les plus motivés par la question, à savoir Veller et Capitaine, ont participé à la deuxième fédération de Pontivy. Si le premier a été un des douze commissaires chargés d'élaborer le mémoire abolitionniste, le second est un des rares députés à s'y être publiquement opposé ${ }^{93}$.

Le 6 décembre, les commissaires produisent un long rapport où ils concluent que le domaine congéable n'est pas d'essence féodale et dynamiserait même l'agriculture régionale, qu'il doit donc être maintenu après avoir été débarrassé de ses abus ${ }^{94}$. Dans ses conclusions, le procureur général syndic souligne l'effet paralysant du débat sur le domaine congéable pour les ventes de biens nationaux et demande en conséquence que les administrateurs, sans prendre position, sollicitent une loi qui lève rapidement l'hypothèque :

"Quel est l'acquéreur qui voudrait nourir les risques d'un changement qui lui deviendrait aussi préjudiciable? il me parait donc indispensable que

en 1829. Michel de Créac'hquérault de Créchenglaz : né en 1742; capitaine au régiment des chasseurs du Gévaudan et chevalier de Saint Louis; électeur en 1790, puis administrateur du Finistère (membre du directoire); il retrouve son régiment en 1791; siège dans la municipalité de Quimper en l'an II; entre au directoire du département en 1795; maire de Penhars en 1801. Jacques René Arnoult : avocat à Pont-l'Abbé; membre de la municipalité d'Ancien Régime; député de paroisse en 1789, puis membre du Comité permanent de sa ville; en 1790, il est élu maire, puis électeur, puis administrateur départemental; agent national sous la Terreur; notaire en 1801 ; siège au conseil d'arrondissement en 1818.

89. Arch. dép. du Finistère, 3 L 1.

90. Bonaventure Auguste René Capitaine du Boisdaniel : avocat; procureur syndic de la municipalité de Quimperlé sous l'Ancien Régime; élu maire de sa ville, puis électeur, puis procureur général syndic du département (après avoir été nommé administrateur) en $1790 ;$ décède en 1792 .

91. Arch. dép. du Finistère, 3 L 1.

92. Veller et Arnoult : cf. supra. Anne Jacques Bernard François Carquet : né en 1733; procureur du roi à Châteauneuf-du-Faou; député de la sénéchaussée de Châteauneuf en 1789 (est élu député suppléant pour les États généraux); nommé électeur, administrateur départemental, puis juge du tribunal du district de Carhaix en 1790; décède en 1793. Nicolas Jacques Cosson de Kervodies : sénéchal de Lesneven; élu officier municipal de sa ville, puis électeur de son canton, puis administrateur départemental (siège au directoire), et, pour finir, juge suppléant du tribunal du district de Lesneven en 1790; élu accusateur public du tribunal criminel du Finistère en 1791 (abandonne rapidement cette fonction et se retire à Lorient).

93. Trevedy, Jean, Les deux fédérations de Pontivy, janvier et février 1790, Vannes et Rennes, 1895, p. 46.

94. Dubreuil, Léon, op. cit., p. 233-243. 
l'Assemblée nationale se décide sur la question de savoir si les rentes domaniales seront ou non, comme toutes autres rentes foncières, franchissables et à quel denier elles le seront. C'est à elle qu'il appartient de régler ce qui peut convenir au bien public; si elle envisage le domaine congéable comme un régime oppressif elle le supprimera, si au contraire il sera conservé ${ }^{95}$."

Le conseil général décide donc de ne proposer aucun projet de décret, se borne à transmettre aux députés ce rapport et à écrire par l'intermédiaire du directoire au comité d'aliénation pour qu'il sollicite de la Constituante une loi $^{96}$.

Les administrateurs départementaux sont informés de l'affaire de Botsorhel le 11 décembre. Sur proposition de la commission du domaine congéable, le conseil général arrête :

"Considérant que si l'infraction aux lois est répréhensible dans un particulier, on ne sauroit la tolérer de la part de corps établis pour les faire observer, considérant que les officiers municipaux de Botsorhel ont formellement contrevenu aux décrets de l'assemblée nationale, notamment à celui du 15 mars en s'opposant à l'exercice du congément du lieu du Rhun; a arrêté et arrête d'improuver la conduite tenue par la municipalité de Botsorhel; arrête en outre qu'elle sera mandatée en la personne de son maire pour rendre compte de sa conduite ${ }^{97}$."

Le 22 décembre, le procureur général syndic inquiet de la tournure que prend le débat sur le domaine congéable dans les campagnes finistériennes, et plus spécialement dans celles du district de Morlaix, renouvelle son vœu d'un appel à l'Assemblée nationale afin qu'elle accélère l'élaboration d'une loi :

"Nous attendons avec impatience un décret qui fixe irrévocablement les droits des propriétaires et des colons; renouvellons nos instances pour qu'une loi sage prévienne au plutot les malheurs dont nous menace la fermentation qui regne dans nos campagnes ${ }^{98}$."

L'assemblée nationale mettra quelques mois pour satisfaire les attentes des administrateurs du Finistère par la loi du 26 août 1791 maintenant le domaine congéable légèrement toiletté. Entre ces deux moments la mobilisation convenancière aura nettement faibli, du moins en Finistère comme le prouve l'absence de réaction de la population de Botsorhel lors de la troisième tentative de congément. Cela ne signifie nullement que la détermination des domaniers est devenue moindre, elle est simplement devenue plus sourde.

Bien évidemment le congément survenu à Botsorhel ne constitue pas un événement majeur de la Révolution en Bretagne. C'est un simple fait divers qui, de par sa nature et par son ampleur, a rencontré un peu plus

95. Arch. dép. du Finistère, 10 L 227.

96. Arch. dép. du Finistère, 3 L 1 et 7 L 1.

97. Arch. dép. du Finistère, 1 Q 931. et 3 L 1.

98. Arch. dép. du Finistère, 10 L 227. 
d'écho qu'un autre. Mais c'est un fait divers signifiant sur le plan social, utilisé pour cette raison à la fois comme révélateur et comme fil conducteur. Quels enseignements peuvent être tirés de l'enquête quant à la réception du domaine congéable à la fin du XVIII ${ }^{\mathrm{e}}$ siècle d'une part, au combat des domaniers au début de la période révolutionnaire de l'autre?

Par un de ces effets dialectiques dont l'histoire est friande, le domaine congéable, après avoir sécrété une société de petits et moyens paysans presque indépendants, et permis à certains de s'enrichir, se trouve rejeté par ceux-la même qu'il a d'une certaine manière promus. Non pas que le système soit particulièrement oppressif, il se serait même adouci comme en témoigne la faiblesse du prélèvement et la rareté des congéments. Mais c'est justement l'état presque résiduel de cette dernière pratique qui le rend désormais si impopulaire de la part de paysans aspirant à la pleine et entière propriété de leurs droits, perspective qui sans doute n'avait jamais été aussi palpable ${ }^{99}$. Ceci sans vouloir nier la complexité des rapports sociaux : les domaniers s'entre-congédient; les plus aisés d'entre-eux souslouent à des conditions parfois peu scrupuleuses; fabriciens, ils gèrent les convenants paroissiaux en fonciers intransigeants.

Suite à un processus de radicalisation, la mobilisation convenancière atteint un pic durant le dernier trimestre de l'année 1790 (un peu avant pour le département des Côtes-du-Nord où la pétition Huchet-Labat fait l'effet d'une bombe). La partie du territoire finistérien la plus en pointe dans le mouvement est incontestablement le district de Morlaix (la précocité de la protestation convenancière dans le Trégor et la proximité du département des Côtes-du-Nord, avec une partie duquel ce district reste culturellement et économiquement lié, l'expliquent). L'information circule intensément dans les campagnes, tant celle qui émane de Paris que celle provenant des départements et des districts voisins. Elle est abondamment discutée, interprétée, puis amendée ou rejetée par les communes et les cantons qui se l'échangent, qui se rencontrent, tissant ainsi de véritables réseaux, particulièrement actifs à l'approche d'échéances importantes (élections, réunion du conseil général du département) ou du fait de sollicitations jugées intéressantes pour la cause (correspondance de députés, pétition Huchet-Labat). Les nouvelles municipalités rurales élues en 1790, peuplées par l'élite paysanne, jouent un rôle essentiel dans cette dynamique $^{100}$. Lors de cette campagne, la paysannerie trouve dans des personnages comme Le Coursonnois des alliés au sein de la bourgeoisie patriote. Cette dernière, unanime sur la nécessité de maintenir l'ordre, est cependant divisée sur la question du domaine congéable comme en

99. Idée empruntée à François FuRET à propos de la féodalité en général, dans $L a$ Révolution française. De Turgot à Napoléon, Paris, 1988, p. 34 (éd. de poche).

100. ADO, Anatoli, op. cit., p. 192-195, constate le même phénomène pour l'ensemble du pays au début de la Révolution. Voir aussi le très beau livre d'Hubert DELPONT, La Victoire des croquants. Les révoltes paysannes du Grand Sud-Ouest pendant la Révolution (17891799), Nérac, 2002. 
témoigne la prise de position des diverses administrations. Celles du Finistère, à l'exception du district de Quimper, semblent largement en retrait comparé à celles des deux autres départements bas-bretons. La paysannerie elle-même ne constitue pas un front de classe uni : certains de ses membres, en particulier les plus riches, n'hésitent pas à recourir pour leur propre intérêt aux pratiques convenancières les plus décriées, y compris après le déclenchement de la Révolution.

En guise d'épilogue, il serait intéressant de revenir sur le sort des victimes du congément du Rhun, Laurent Le Guilloux et Anne Cloarec. En l'an III on les retrouve employés comme simples journaliers sur la métairie du Foënnec, devenue bien d'émigré ${ }^{101}$. En " pinagotant ${ }^{102}$ " un peu, on peut donc imaginer que leur expulsion a été synonyme de déclassement social.

\section{RESUME}

Contrairement à nombre de paysans français, les domaniers bas-bretons ont sauf quelques rares cas, privilégié l'action légale dans leur combat pour la possession de la terre durant la Révolution. Parmi les exceptions figure une affaire de congément survenue dans la paroisse de Botsorhel à la fin de l'année 1790. Ce congément ne constitue nullement un événement majeur de la Révolution en Bretagne, c'est un simple fait divers. À l'examen pourtant, ce dernier se révèle socialement très signifiant. Aussi, en suivant ce fil conducteur, l'article se propose d'étudier dans le cadre du Finistère et plus spécialement du district de Morlaix, l'évaluation du système convenancier par ses utilisateurs à la fin de l'Ancien Régime, les débuts du mouvement domanier, les positions adoptées en réponse par les nouvelles autorités judiciaires et administratives.

\section{ABSTRACT}

Unlike many French peasants, the tenants of domaine congéable in Lower Brittany, with the exception of a few cases, favoured the legal action in their fight for land possession during the Revolution. Among these exceptions there was the stormy eviction of tenants in the Botsorhel parish in the late 1790. This eviction was not a major moment of Revolution in Brittany, it was a trivial event. However, it rapidly proved to me to be socially interesting. Following this vital lead, I propose to study the evaluation of domaine congéable system by the tenants at the end of Old Regime, the beginnings of their protest movement and the answer of the new judicial and administrative authorities, all these within Finistère and especially the district of Morlaix.

101. Arch. dép. du Finistère, 1 Q 931.

102. CoRBIN, Alain, Le Monde retrouvé de Louis-François Pinagot. Sur les traces d'un inconnu 1798-1876, Paris, 1998. 
